\title{
Evaluation of intramolecular charge transfer state of 4- $N, N$-dimethylamino cinnamaldehyde using time-dependent density functional theory
}

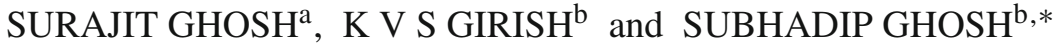 \\ ${ }^{a}$ Department of Physics and Technophysics, Vidyasagar University, Midnapore 721 102, India \\ ${ }^{\mathrm{b}}$ School of Chemical Sciences, National Institute of Science Education and Research, \\ Bhubaneswar, 751 005, India \\ e-mail:sghosh@niser.ac.in
}

MS received 30 November 2012; revised 28 February 2013; accepted 5 April 2013

\begin{abstract}
Intramolecular charge transfer of 4-N,N-dimethylamino cinnamaldehyde (DMACA) in vacuum and in five different aprotic solvents has been studied by using time-dependent density functional theory (TDDFT). Polarizable continuum model (PCM) was employed to consider solvent-solute interactions. The potential energy curves were constructed at different torsional angle of $N, N$-dimethylamino moiety with respect to the adjacent phenyl ring. A large bathochromic shift in our calculated emission and absorption energies for polar solvents is a clear reminiscent of charge transfer nature of the excited state. Finally, the reported results are in agreement with experimental findings.
\end{abstract}

Keywords. Twisted intermediate charge transfer (TICT); density functional theory (DFT); dimethylamino cinnamaldehyde (DMACA); Gaussian.

\section{Introduction}

The nature of charge transfer (CT) state within a molecule remains obscure despite decades of experimental and theoretical research. ${ }^{1-18} \quad 4-N, N$ dimethylamino benzonitrile (DMABN) molecule with an inherent donor and an acceptor group exhibits dualemission feature in its emission spectrum. ${ }^{1}$ Grabowski explained this phenomenon by proposing a twisted intermediate charge transfer (TICT) model. ${ }^{2}$ Evidence suggests that the intramolecular TICT process from a donor to an acceptor could be achieved by a twisting motion of the donor moiety that promotes initially generated locally excited (LE) state to an energetically relaxed charge transfer state $(\mathrm{CT}){ }^{2-6,8,13}$ Besides TICT model, a planner intramolecular charge transfer (PICT) and wagged intramolecular charge transfer (WICT) model have also been adopted by some other groups. ${ }^{5,6}$

Dual-fluorescence of a molecule carrying an inherent donor and acceptor is strongly influenced by the electronic energy gap of first two excited states $\mathrm{n} \pi *(\mathrm{HOMO}-1$ to LUMO, HOMO-highest occupied molecular orbital, LUMO-lowest unoccupied molecular orbital) and $\pi \pi^{*}($ HOMO to LUMO). These

*For correspondence electronic excited states are modified either by a mild change in donor strength or by the solvent polarity. In non-polar aprotic solvent the energy gap between $\mathrm{n} \pi *$ (LE) and $\pi \pi^{*}(\mathrm{CT})$ state is relatively smaller compared to that in polar solvent. In polar aprotic solvent, more stable CT (highly polar) state dominates over $n \pi *$ (less polar, LE state) in S1 state (first excited singlet state).

This paper investigates the nature of CT state of DMACA in a series of solvents with fairly diverse range of polarity. The impressive success of density functional theory (DFT) inspired us to employ this method for examination of the small energy gap within the lower excited states (S1-S2) of DMACA. ${ }^{15-20}$ From our study, it is evident that a remarkable red shift in the fluorescence maxima of DMACA in polar solvent is subject to a strong intramolecular charge transfer involving in the S1 state.

\section{Computational methods}

Ground state optimizations of DMACA (scheme 1) was performed by using B3LYP hybrid function with 6-31G (d) basis set at DFT level. ${ }^{15-17}$ Several other authors also used similar basis set to optimize the ground state geometry of a small molecule. ${ }^{8,16}$ A rigorous formalism (time-dependent density functional theory, 


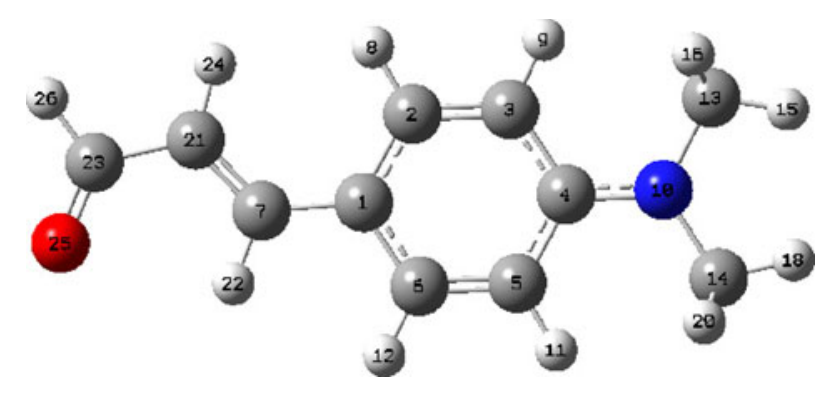

Scheme 1. Optimized geometry of DMACA in the ground electronic state.

TDDFT) was used for transition energy calculation within the DFT framework using 6-31G+(d, p) basic set. ${ }^{15-18,21-23}$ Solvation energy was calculated using time dependent density functional theory-polarizable continuum model (TDDFT-PCM). ${ }^{19,20}$ Polarizable continuum model (PCM) is frequently used in computational chemistry to investigate the delicate solventsolute interactions within the solvation shell. In this model solvents, instead as individual molecules, are considered as polarizable and continuum. According to this model, the molecular free energy $\left(\mathrm{G}_{\mathrm{sol}}\right)$ of solvation can be obtained from the following equation ${ }^{20 b}$

$$
\mathrm{G}_{\mathrm{sol}}=\mathrm{G}_{\mathrm{es}}+\mathrm{G}_{\mathrm{dr}}+\mathrm{G}_{\mathrm{cav}},
$$

where, $\mathrm{G}_{\mathrm{es}}=$ electrostatic energy, $\mathrm{G}_{\mathrm{dr}}=$ dispersionrepulsion energy and $\mathrm{G}_{\text {cav }}=$ cavitation energy.

In this work, by using PCM model we have calculated the solvent induced stabilization energy at different energy levels of DMACA within the DFT framework. All calculations were performed using Gaussian $03 \mathrm{w}$ package. ${ }^{24}$ In analogy to some recent works, we have considered the optimized structure obtained in vacuum to hold good for the solution phase as well as for the excited state calculations assuming not much change in the corresponding geometries. ${ }^{16-18}$ Absorption energy obtained from TDDFT calculation is the vertical excitation energy without zero point correction. Potential energy surface (PES) of DMACA was obtained by pursuing an intramolecular rotation along twist coordinate at the donor side within the DFT framework. The details of twisting motions are described in section 3.2.

\section{Results and discussion}

\subsection{Ground state properties and vertical excited states of DMACA}

Ground state optimized structure of DMACA is planar, and almost without any twisting between $N, N$ dimethylamino group to the phenyl moiety (scheme 1). The ground state dipole-moment of DMACA in vacuum was found to be 7.4 D. However, we have detected a larger ground state dipole-moment in polar aprotic solvent compared to that observed in vacuum. This is because the polar solvent always stabilizes the dipolar state of a molecule by dipole-dipole interactions.

In this section, we will try to address the absorption energy (vertical energy) of DMACA in vacuum and in different aprotic solvents with fairly diverse range of polarity. We did not consider protic solvent in order to avoid the complication that arises in the excited state on account of hydrogen bond formation. ${ }^{16-18}$ An analysis of TDDFT wave function in vacuum shows

Table 1. Computed excited state parameters and absorption energy of DMACA in vacuum and other aprotic solvents using TDDFT method with B3LYP hybrid function.

\begin{tabular}{lcccc}
\hline Medium/solvent & State & $\begin{array}{c}\text { Oscillator } \\
\text { strengths }(\mathrm{f})\end{array}$ & $\begin{array}{c}\text { Computed absorption } \\
\text { energies }(\Delta \mathrm{E}, \mathrm{eV})\end{array}$ & $\begin{array}{c}\text { Experimental absorption } \\
\text { energies }(\Delta \mathrm{E}, \mathrm{eV})\end{array}$ \\
\hline Vacuum & $\mathrm{S}_{1}(\mathrm{LE})$ & 0.0001 & 3.34 & \\
Heptane & $\mathrm{S}_{2}(\mathrm{CT})$ & 0.7672 & 3.42 & $3.55^{\mathrm{b}}$ \\
& $\mathrm{S}_{1}(\mathrm{CT})$ & 0.932 & 3.19 & $3.40^{\mathrm{c}}$ \\
Benzene & $\mathrm{S}_{2}(\mathrm{LE})$ & 0.0001 & 3.36 & $3.43^{\mathrm{c}}$ \\
Toluene & $\mathrm{S}_{1}(\mathrm{CT})$ & 0.9581 & 3.14 & $3.47^{\mathrm{c}}$ \\
\multirow{2}{*}{ THF } & $\mathrm{S}_{2}(\mathrm{LE})$ & 0.0001 & 3.38 & 3.14 \\
& $\mathrm{~S}_{1}(\mathrm{CT})$ & 0.958 & 3.39 & $3.41^{\mathrm{c}}$ \\
ACN & $\mathrm{S}_{2}(\mathrm{LE})$ & 0.0001 & 3.09 & \\
& $\mathrm{~S}_{1}(\mathrm{CT})$ & 0.9479 & 3.50 & \\
\hline
\end{tabular}

${ }^{\mathrm{a}}$ Basis set 6-31G+(d, p)

${ }^{b}$ Values taken from Ref. ${ }^{9}$

${ }^{\mathrm{c}}$ Values taken from Ref. ${ }^{7}$ 


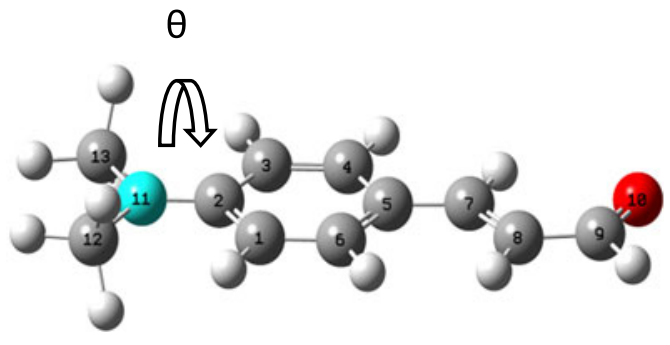

LE state of DMACA

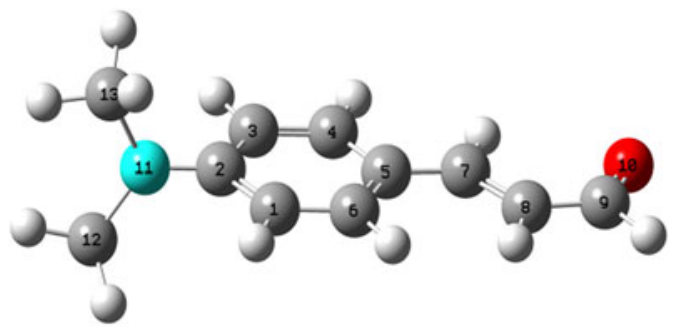

TICT state of DMACA

Scheme 2. Twisting of DMACA in the excited state.

that the $\mathrm{S} 1$ and $\mathrm{S} 2$ states are mainly composed of $\mathrm{n} \pi *$ (HOMO-1 to LUMO; HOMO - highest occupied molecular orbital, LUMO - lowest unoccupied molecular orbital) and $\pi \pi^{*}$ (HOMO to LUMO) nature of transition. In vacuum, the energy gap between these two states is significantly small (table 1) and the polar S2 (CT) state has larger oscillator strength with predominantly $\pi \pi^{*}$ (HOMO to LUMO) transition character (table 1). However, lack of any experimental data on absorption energy of DMACA in vacuum has left our computational data unverified. In the presence of polar solvent, the highly polar charge transfer state is significantly stabilized by dipole-dipole interactions and switch over to the first excited state (S1). With increase in solvent polarity, the energy gap between LE and CT state increases quite drastically compared to the vacuum. In polar solvent, using similar kind of TICT probe (Michler's ketone) Mondal et al. ${ }^{13}$ observed an additional low intensity band at the blue end $(\sim 320 \mathrm{~nm})$ in the absorption spectrum of Michler's ketone that becomes more prominent and well separated from the high intensity one with increase in solvent polarity. Note, the low intensity band originates from a forbidden (HOMO-1 to LUMO) transition. The position of the band hardly shows any solvent polarity dependence due to its weakly polar nature in the excited state. ${ }^{13}$ Besides vacuum, we have also performed our DFT/TDDFT calculations on a series of aprotic solvents with different polarity. Our calculated results in aprotic solvents nicely follow the experimental values reported by Singh et $a .^{7}$ and Panja et al., ${ }^{9}$ which is a strong support towards the reliability of our computed values and also appropriate choice of basis sets (table 1).

\subsection{Twisting dynamics and emission property in vacuum and in aprotic solvents}

In this section, we will try to correlate the relaxation process of DMACA by considering a twisting motion of the donor moiety relative to the adjacent phenyl moiety.
In brief, after photo excitation, $N, N$-dimethylamino moiety of DMACA in polar aprotic solvent exhibits a $90^{\circ}$ twisting with respect to the phenyl ring. Such kind of twisting motion is a prerequisite condition for the relaxation of TICT state. In this section we will discuss the potential energy curve via twisting motion of the donor moiety (scheme 2). The twisting angle is denoted by the torsional angle ' $\theta$ ', to describe the twisting motion of the $N, N$-dimethylamino group relative to the phenyl ring. In scheme $1, \theta$ represents the dihedral angle $<13-11-2-3$. Ground state optimized structure of DMACA is planer; dihedral angle $(\theta)$ between $N, N$-dimethylamino group and the phenyl ring (to which it is attached) is $\sim 0^{\circ}$ (scheme 1). During PES calculation, for every electronic states, all geometrical parameters are held fixed to that optimized for the ground state; except the twisting angle $(\theta)$, which is varied $10^{\circ}$ in each steps. A torsional motion of the $\mathrm{N}, \mathrm{N}$-dimethylamino group to an angle of $90^{\circ}$ is essential to yield a minimum on the excited state energy surface. PES of DMACA in vacuum is depicted in

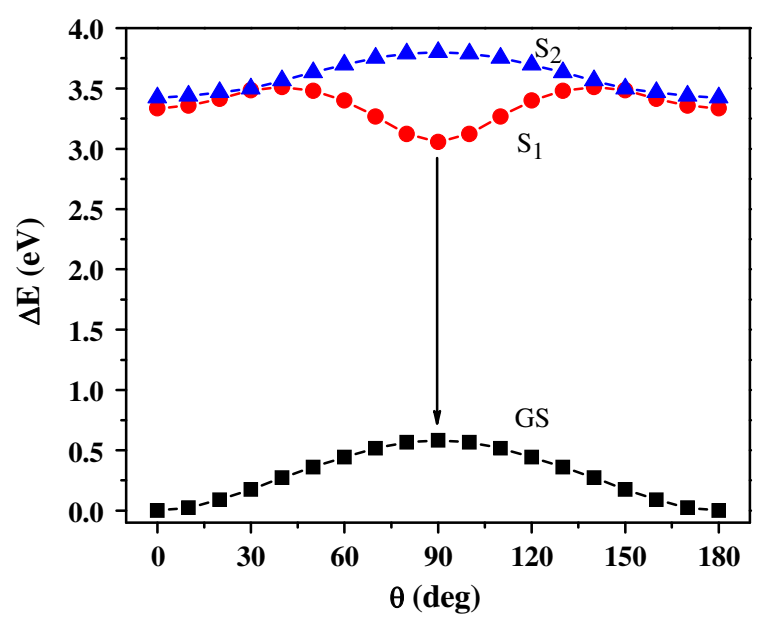

Figure 1. Potential energy surface (PES) of DMACA in vacuum for the ground state (GS) and first two excited states $\left(\mathrm{S}_{1}\right.$ and $\left.\mathrm{S}_{2}\right)$ along the twist coordinate of the donor moiety; $\theta$ describe the rotation of $N, N$-dimethylamino group. 


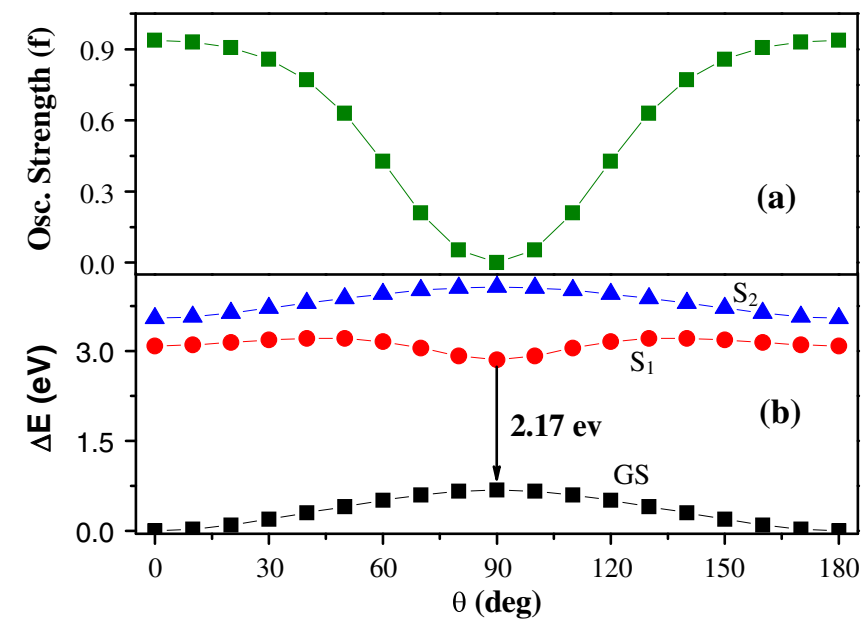

Figure 2. (a) Plot of oscillator strength of $S_{1}$ state for DMACA along the twisting coordinate of the donor moiety in acetonitrile solvent. (b) Potential energy surface (PES) of DMACA for the ground state (GS) and first two excited states $\left(\mathrm{S}_{1}\right.$ and $\left.\mathrm{S}_{2}\right)$ along the twist coordinate of donor moiety in acetonitrile. $\theta$ describe the rotation of $N, N$-dimethylamino group.

figure 1 , whereas figure $2 \mathrm{a}, \mathrm{b}$, respectively show the oscillator strength and PES of DMACA in a polar aprotic solvent (acetonitrile, ACN). Besides vacuum and acetonitrile, we have also calculated the PES in some other solvents with different polarity, such as heptane, benzene, toluene, and tetrahydrofuran (THF). In both vacuum and solvent medium the ground state energy gradually increases along the twist coordinate and reaches a maximum at $\theta \sim 90^{\circ}$. On the contrary, the energy of CT state decreases in the same direction and ultimately reaches to a local energy minimum at $\theta \sim 90^{\circ}$; resulting a smallest energy gap between the ground and the first excited state $\left(\mathrm{S}_{1}\right)$. A red-shifted emission and lowering of oscillator strength along the donor twist coordinate is a clear reminiscent of decreasing the vertical transition energy $\left(\Delta \mathrm{E}_{1 \mathrm{~s}}\right)$ and increasing of $n \pi^{*}$ character of the emitting species (figures 1 and $2)$. In the ground state optimized geometry, predominantly $\pi \pi^{*}$ nature of transition (HOMO to LUMO) is observed and the donor lone pair is delocalized all over to the adjacent phenyl group (figure $3 a, b$ ). However, the twisted conformer $\left(\theta \sim 90^{\circ}\right)$ carries predominately $\mathrm{n} \pi *$ transition character (HOMO-1 to LUMO) with localized donor lone pair which becomes available for charge transfer to the $\pi^{*}$ orbital of the acceptor $(\mathrm{C}=\mathrm{O})$ moiety (scheme 2, figure $3 \mathrm{c}, \mathrm{d}$ ). This is a clear feature of charge transfer that takes place when donor group becomes orthogonal to the accepter group, i.e., decoupling is maximum.

In table 2 we have compared our computed emission energies with the corresponding reported experimental values. ${ }^{7}$ In all the solvents mentioned, our calculated values are quite higher compared to the experimental values. Recently some other groups have also reported a higher computed emission energy obtained from their PES calculation. ${ }^{16-18}$ The discrepancy between the calculated values and the experimental results may be

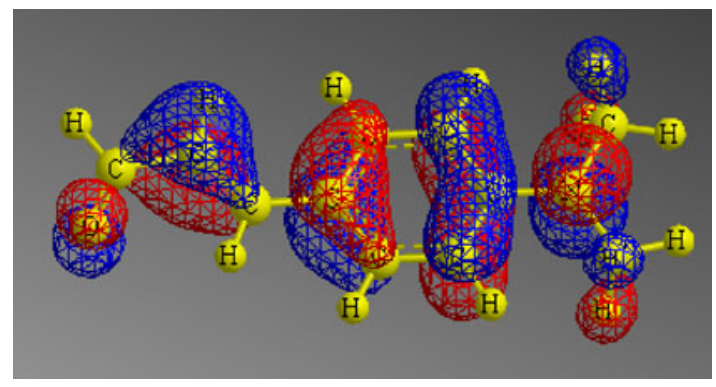

(a)

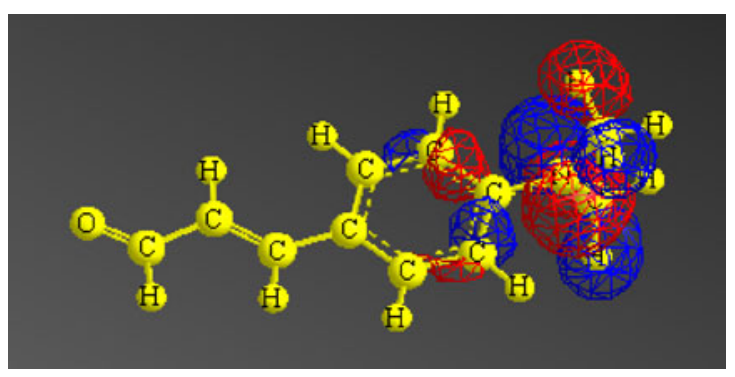

(c)

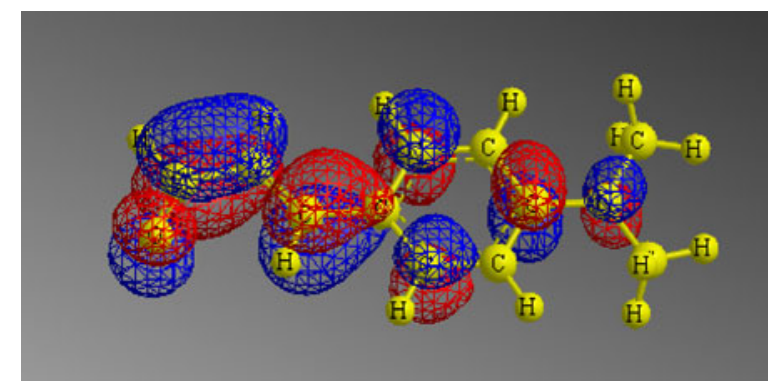

(b)

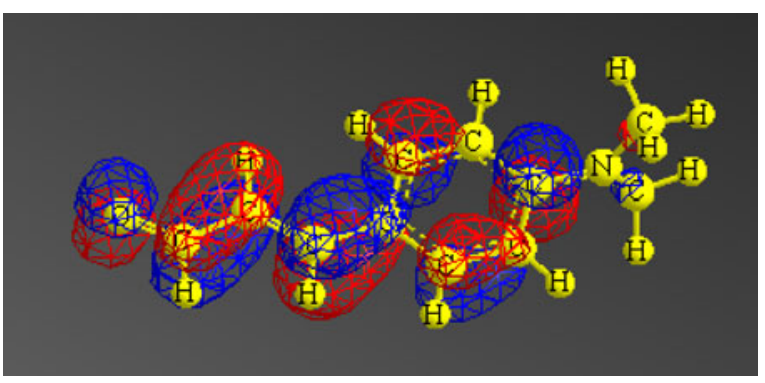

(d)

Figure 3. (a) HOMO and (b) LUMO of planar ground state of DMACA; (c) HOMO and (d) LUMO of twisted ground state of DMACA. 
Table 2. Computed excited state parameters and emission energies of DMACA in vacuum and other aprotic solvents using DFT and TDDFT method with B3LYP hybrid function.

\begin{tabular}{|c|c|c|c|c|c|}
\hline Medium/solvent ${ }^{\mathrm{a}}$ & $\varepsilon^{\mathrm{b}}$ & State & $\begin{array}{l}\text { Oscillator } \\
\text { strengths (f) }\end{array}$ & $\begin{array}{l}\text { Computed emission } \\
\text { energies }(\Delta \mathrm{E}, \mathrm{eV})\end{array}$ & $\begin{array}{l}\text { Experimental emission } \\
\text { energies }(\Delta \mathrm{E}, \mathrm{eV})^{\mathrm{c}}\end{array}$ \\
\hline Vacuum & & $\mathrm{S}_{1}(\mathrm{CT})$ & 0.00 & 2.4739 & No reported value was found \\
\hline Heptane & 1.92 & $\mathrm{~S}_{1}(\mathrm{CT})$ & 0.00 & 2.3289 & No TICT emission reported \\
\hline Benzene & 2.3 & $\mathrm{~S}_{1}(\mathrm{CT})$ & 0.00 & 2.31 & 2.98 \\
\hline Toluene & 2.38 & $\mathrm{~S}_{1}(\mathrm{CT})$ & 0.00 & 2.3035 & 3.00 \\
\hline $\mathrm{THF}$ & 7.5 & $\mathrm{~S}_{1}(\mathrm{CT})$ & 0.00 & 2.2150 & 2.97 \\
\hline $\mathrm{ACN}$ & 37.5 & $\mathrm{~S}_{1}(\mathrm{CT})$ & 0.00 & 2.1743 & 2.79 \\
\hline $\mathrm{ACN}^{\mathrm{d}}$ & 37.5 & $\mathrm{~S}_{1}(\mathrm{CT})$ & 0.00 & 2.34 & \\
\hline
\end{tabular}

${ }^{\mathrm{a}}$ Basis set $6-31 \mathrm{G}+(\mathrm{d}, \mathrm{p})$

${ }^{\mathrm{b}}$ Static dielectric constant; values taken from Ref. ${ }^{25,26}$

${ }^{\mathrm{c}}$ Values taken from Ref. ${ }^{7}$

${ }^{\mathrm{d}}$ Ground state optimization was performed in acetone solvent (instead of taking ground state optimized structure in vacuum and applied it to the solvent phase assuming not much change in geometry). More details have been provided in section 3.2 (last paragraph)

attributed to the non-optimized excited state geometry of DMACA or may be due to some missing parameters within the analytical gradient of TDDFT method. Also, we used the ground state optimized structure in vacuum and applied it to the solvent phase assuming not much change in geometry. ${ }^{16-18}$ We have tested the reliability of this assumption by comparing energy levels of these vacuum optimized structures in organic solvent (assuming not change much in molecular geometry) with those, when optimizations were carried out by the solvent itself. We performed this calculation at least for one solvent (acetonitrile), to make it certain that the significant discrepancy in emission energy (discrepancies between our data with the experimentally reported value) is not due to the slight non-ideality in ground state optimized structure. Ground state optimization of twisted structure (keeping twist angle fixed at $90^{\circ}$ angle, rest part was allowed to relax) and excited state calculations of DMACA in acetonitrile solvent were performed within DFT and TDDFT framework [with $6-31+\mathrm{G}(\mathrm{d}, \mathrm{p})$ basis set]. The vertical excitation energy (from $S_{0}$ to $S_{1}$ ) of this acetonitrile optimized structure was found to be $2.34 \mathrm{eV}$, which provides little improvement towards achieving the experimental value (2.79 eV, table 2) compared to that $(2.17 \mathrm{eV}$, table 2) obtained from the vacuum optimized structure (with assumption not change much in geometry within the solvent). Till now, the excited state geometry change during TICT process has been remained under investigations. Excited state optimization with Gaussian 03 is computationally very expensive and we were unable to do it. However, some other molecular level calculation with accessible analytical gradients (like, TURBOMOLE) might provide us an easy characterization of the excited state geometry. Therefore, we cannot rule out the discrepancy between our computed emission energies with experimental values are not due to the small geometrical change in the excited state geometry.

\section{Conclusion}

In this work, using TDDFT method we have calculated the absorption and emission energies of DMACA in vacuum and in five aprotic solvents with different static polarity. From our study it may be concluded that after photo excitation of DMACA, it undergoes an intramolecular twisting motion of the $N, N$ dimethylamino group to yield a minimum on the energy surface curve. This type of twisting leads to an excited state relaxation from LE to a TICT state. In vacuum, highly polar CT state becomes less stable compared to less polar LE state and resides to an energetically high S2 state. However, the polar solvent stabilizes the CT state to a greater magnitude compared to the LE state that inverts the CT state to an energetically more stable $\mathrm{S} 1$ state, which is responsible for the observed red shift in the calculated emission and absorption energy in polar solvent compared to the non-polar solvent.

\section{Acknowledgements}

Surajit Ghosh thanks the funding support from the Department of Science and Technology (DST), New Delhi, India via grant SR/FTP/PS.066/2010 and the University Grants Commission (UGC), New Delhi, India for Special Assistance. Subhadip Ghosh also thanks Ramanujan Fellowship Grant, DST, for the support to carry out this work. We thank Dr. Himanshu S Biswal for many fruitful and stimulated discussions. 


\section{References}

1. Lippert E, Luder W and Boos H 1962 In Advances in molecular spectroscopy, A Mangini (ed.) (Oxford: Pergamon) p. 443

2. Rotkiewicz K, Grellmann K H and Grabowski Z R 1973 Chem. Phys. Lett. 19315

3. Zachariasse K A, Grobye M and Tauer E 1997 Chem. Phys. Lett. 274372

4. Zachariasse K A, Haar T V D, Hebecker A, Leinhos U and Kuhnle W 1993 Pure Appl. Chem. 651745

5. Schuddeboom W, Jonker S A, Warman J M, Leinhos U, Kuhnle W and Zachariasse K A 1992 J. Phys. Chem. 96 10809

6. Sobolewski A L, Sudholt W and Domcke W 1998 J. Phys. Chem. A 1022716

7. Singh T S, Moyon N S and Mitra S 2009 Spectrochim. Acta Part A $\mathbf{7 3} 630$

8. Pal T, Paul M and Ghosh S 2008 J. Mol. Struct. (THEOCHEM) 8608

9. Panja S and Chakravorti S 2003 Chem. Phys. Lett. 367 330

10. Veldhoven E V, Zhang H, Rettig W, Brown R G, Hepworth J D and Glasbeek M 2002 Chem. Phys. Lett. 363189

11. Glasbeek M and Zhang H 2004 Chem. Rev. 1041929

12. Bangal P R, Panja S and Chakravorti S 2001 J. Photochem. Photobiol. A: Chemistry 1395
13. Mondal J A, Ghosh H N, Ghanty T K, Mukherjee T and Palit D K 2006 J. Phys. Chem. A 1103432

14. Singh A K, Ramakrishna G, Ghosh H N and Palit D K 2004 J. Phys. Chem. A 1082583

15. Ramasami P 2006 J. Mol. Struc. (THEOCHEM) 767 19

16. Cave R J and Castner E W Jr 2002 J. Phys. Chem. A 106 12117

17. Duan X-H, Li X-Y, He R-X and Cheng X-M 2005 J. Chem. Phys. 122084314

18. Chakraborty A, Kar S, Nath D N and Guchihait N 2007 J. Chem. Sci. 119195

19. Cossi M, Barone V, Cammi R and Tomasi J 1996 Chem. Phys. Lett. 255327

20. (a) Miertus S, Scrocco E and Tomasi J 1981 Chem. Phys. 55 117; (b) Tomasi J, Mennucci B and Cammi R 2005 Chem. Rev. 1052999

21. Runge E and Gross E K U 1984 Phys. Rev. Lett. 52997

22. Casida M E 1996 In Recent developments and applications of modern density functional theory. J M Seminario (ed.) (Amsterdam: Elsevier) p. 391

23. Pandit S, De D and De B R 2006 J. Mol. Struct. (THEOCHEM) $\mathbf{7 7 8} 1$

24. Frisch M J, Trucks G W, Schlegel H B, Scuseria G E and Robb A et al. 2004 Gaussian Inc. Wallingford, CT

25. Dean J A 1979 In Lange's handbook of chemistry (New York: McGrawHill)

26. http://en.wikipedia.org/wiki/Solvent 